\title{
Preparation and electrical properties of polyimide/carbon nanotubes composites
}

\author{
ASEEL A. KAREEM* \\ Department of Physics, College of Science, University of Baghdad
}

\begin{abstract}
Polyimide/MWCNTs nanocomposites have been fabricated by solution mixing process. In the present study, we have investigated electrical conductivity and dielectric properties of PI/MWCNT nanocomposites in frequency range of $1 \mathrm{kHz}$ to $100 \mathrm{kHz}$ at different MWCNTs concentrations from $0 \mathrm{wt} . \%$ to $15 \mathrm{wt} . \%$. It has been observed that the electrical conductivity and dielectric constants are enhanced significantly by several orders of magnitude up to $15 \mathrm{wt} \%$ of MWCNTs content. The electrical conductivity increases as the frequency is increased, which can be attributed to high dislocation density near the interface. The rapid increase in the dielectric constant at a high MWCNTs content can be explained by the formation of a percolative path of the conducting network through the sample for a concentration corresponding to the percolation threshold. The high dielectric constant at a low frequency $(1 \mathrm{kHz})$ is thought to originate from the space charge polarization mechanism. $\mathrm{I}-\mathrm{V}$ characteristics of these devices indicate a significant increase in current with an increase in multi-walled carbon nanotube concentration in the composites. The SEM images show improved dispersion of MWCNTs in the PI matrix; this is due to the strong interfacial interactions.
\end{abstract}

Keywords: multi-walled carbon nanotubes (MWCNTs); nanocomposites; electrical properties; polyimide

\section{Introduction}

Carbon nanotubes (CNTs) have generated huge activity in many areas of science and engineering due to their extraordinary physical and chemical properties. CNTs possess excellent electrical, mechanical, thermal, magnetic properties, low density, high surface area, and high chemical stability $[1,2]$.

They have extremely low electrical resistance. Resistance occurs when an electron collides with a defect in a crystal structure of a material through which it is passing. The defect could be an impurity atom, an imperfection in the crystal structure, or an atom vibrating about its position in the crystal. Such collisions deflect the electron from its path. But the electrons inside a carbon nanotube are not so easily scattered. Because of their very small diameter and huge length to diameter ratio that can be up in the millions or even higher, so they could be ideal additives for the polymeric nanocomposites [3].

*E-mail: aseelalobaedy@yahoo.com
Polyimide (PI) is one of the high-performance polymers that has been widely used as a dielectric and packaging material for integrated circuits in the microelectronics industry due to its outstanding mechanical, thermal, and dielectric properties [4].

Electrically, polyimide by itself is an insulator and its use in application areas that need electrically conductive polymers has been limited by the fact that accumulation of electrostatic charge at the polymer surface can cause heating of material, which, in turn, can damage the material. Therefore, there is a need to increase the electrical conductivity of the polymer [5].

MWCNTs/polyimide nanocomposite is one of interesting topics of MWCNTs/polymer composites. The use of carbon nanotubes as a conductive and high strength filler in polymer hosts has attracted great attention recently. However, in order to increase the conductivity of the polymer and to reinforce mechanically the polymer structure at low weight fraction of MWCNTs, the integration of the MWCNTs into the polymer matrix has to be effective [6]. 
Park et al. [7] adapted two-step process for preparing PI/MWCNTs composites films containing from 0 wt. $\%$ to $2.0 \mathrm{wt} . \%$ of MWCNTs. At the first step, an amide acid polymer was synthesized and then, carbon nanotubes were added to the prepared polymer and the mixture was then stirred and cast to form films. The results showed that the electrical resistivity of the polyimide/MWNT composites decreased with an increase in the MWNT content.

Mo et al. [8] fabricated polyimide/multi-walled carbon nanotube (PI-MWNT) nanocomposites by in-situ polymerization process. The dielectric constant of the PI-MWNT nanocomposites increased from 3.5 to 80 at $1 \mathrm{kHz}$ as the MWNT content increased to $15 \mathrm{wt} \%$. Jiang et al. [9] studied the electrical properties of PI-MWNT nanocomposites prepared by in-situ polymerization process. The results implied that the percolation threshold for the electric conductivity of the resultant PI-MWNT composite was $0.15 \%$. The electrical conductivity increased by more than 11 orders of magnitude to $10^{-4} \mathrm{~S} / \mathrm{cm}$ at the percolation threshold.

In this study, we demonstrate an effective way to prepare PI/MWCNTs nanocomposites. A new poly(amic acid) (PAA) derived from pyromellitic dianhydride (PDMA) and p-phenylene diamine (PDA) were designed and synthesized as a polyimide matrix to act as dispersant for the MWCNTs in order to study their electrical properties and microstructure.

\section{Experimental}

Initially, $40 \mathrm{~mL}$ of $\mathrm{n}$-methyl-1-pyrrolidinone (NMP) and appropriate amount of commercial multi-walled carbon nanotubes (MWCNTs) obtained from Nanocyl S.A. (Belgium) were taken. The MWCNTs with the diameter of $30 \mathrm{~nm}$ to $60 \mathrm{~nm}$, length of $1.5 \mu \mathrm{m}$ to $400 \mu \mathrm{m}$ and carbon purity $>90 \%$ were placed in a flask and stirred vigorously under ultrasonication for $12 \mathrm{~h}$ at room temperature to obtain a homogeneous suspension.

Then, polyimide was prepared using two different monomers, pyromellitic dianhydride (PMDA) and p-phenylenediamine (PDA), commercially available from Sigma-Aldrich. $2 \mathrm{~g} \mathrm{(10} \mathrm{mmol)} \mathrm{PDA}$ and $40 \mathrm{~mL}$ NMP were placed in a flask and stirred for $30 \mathrm{~min} .2 \mathrm{~g}$ (10 mmol) PMDA were then added to the solution in portions and stirred overnight at room temperature. As a result, $20 \%$ poly(amic acid) (PAA) was obtained. The PI/MWCNTs composite films were prepared by thermal imidization of the PI/MWCNTs cast films. Briefly, a PI solution in NMP at $2.5 \mathrm{wt} \%$ was added to the modified MWCNTs/NMP suspension at $0 \mathrm{wt} . \%, 3 \mathrm{wt} . \%$, 9 wt. $\%$ and 15 wt.\% MWCNTs based on the polymer, respectively. After stirring for several hours, the PI/MWCNTs mixture was cast onto a glass plate and dried under vacuum at $50{ }^{\circ} \mathrm{C}$ for $48 \mathrm{~h}$. For thermal curing of the PI, the cast composite films were heat-treated at $200{ }^{\circ} \mathrm{C}$ for $3 \mathrm{~h}$ followed by $300{ }^{\circ} \mathrm{C}$ for $2 \mathrm{~h}$.

Dielectric parameters such as capacitance, C and the dissipation factor, $\tan \delta$ were measured by using LCR Meter model (HP- 4275) at various frequencies $(1 \mathrm{kHz}$ to $100 \mathrm{kHz})$ at room temperature. The dielectric constants, $\epsilon_{\mathrm{r}}$ were calculated by the equation:

$$
C=\varepsilon_{r} \varepsilon_{0} \frac{A}{d}
$$

where $\epsilon_{0}$ is vacuum permittivity equal to $8.85 \times 10^{-12} \mathrm{~F} / \mathrm{m}$. A $\left[\mathrm{cm}^{2}\right]$ is the electrode area and $\mathrm{d}[\mathrm{cm}]$ is the thickness of the specimen.

AC conductivity $\left(\sigma_{\mathrm{ac}}\right)$ was calculated from the relation:

$$
\sigma_{a . c}=\varepsilon_{o} w \varepsilon_{r} \tan \delta
$$

where, $\tan \delta$ is the dielectric dissipation factor and $\omega$ is the angular frequency.

Current-voltage measurements were performed at room temperature under DC field as a function of 3 wt.\%, 9 wt.\% and 15 wt.\% MWCNTs concentration.

Morphological properties were evaluated by a scanning electron microscope Hitachi, FE-SEM (S-4200). 


\section{Results and discussion}

Fig. 1 shows the AC conductivity as a function of MWCNTs concentration. The general theory explaining the conduction mechanism of MWCNTs filled polymer composites is the "theory of conductive paths", which suggests that the existence of conductive paths is responsible for the conductivity of the composites. With increasing content of a filler, conductive paths among the MWCNTs increase, and the average distance between the MWCNTs becomes smaller; thus, the resistivity of the composites decreases and the electrical conductivity increases $[10,11]$.

The variation in electrical conductivity of the composites for different frequencies is shown in Fig. 1. The electrical conductivity increases as the frequency increases in the range of $1 \mathrm{kHz}$ to $100 \mathrm{kHz}$. The interpretation of this behavior may be that high dislocation density near the interface is basically attributed to the composite materials. Electrical conductivity depends on the number of charge carriers in the bulk of the material, the relaxation time of the charge carriers and the frequency of the applied electric field $[11,12]$.

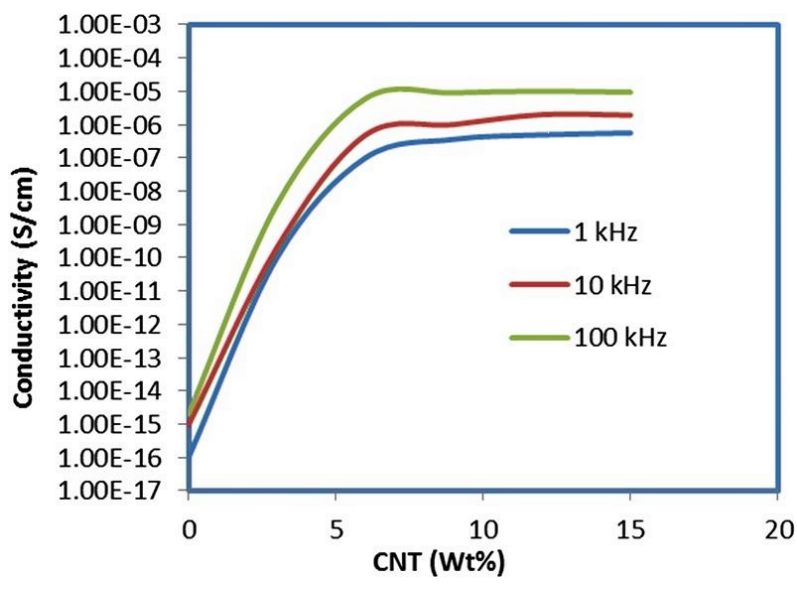

Fig. 1. Electrical conductivity $\sigma$ as a function of MWCNTs content at different frequencies.

The plots of dielectric constant of the composite versus MWCNTs concentration at three different frequencies is shown in Fig. 2. It can be seen from the figure that the dielectric constant of PI/MWCNTs nanocomposites decreases with an increasing frequency. Dielectric constant is a frequency dependent parameter in polymer systems; the dielectric constant is governed by the number of dipoles present in the system and their ability to orient along an applied electric field [12]. Usually, the molecular groups which are attached perpendicular to the longitudinal polymer chain contribute to the dielectric relaxation mechanism. At lower frequencies of applied voltage, the free dipolar functional groups in the polymer chain can orient themselves, which results in a higher dielectric constant value at these frequencies. As the electric field frequency increases, the bigger dipolar groups find it difficult to orient at the same pace as the alternating field, so the contributions of these dipolar groups to the dielectric constant is reduced, resulting in a continuously decreasing dielectric constant of the polymer system at higher frequencies [8]. As shown in the same figure, we can see that the dielectric constant increases as the concentration of MWCNTs in the polymer matrix is increased.

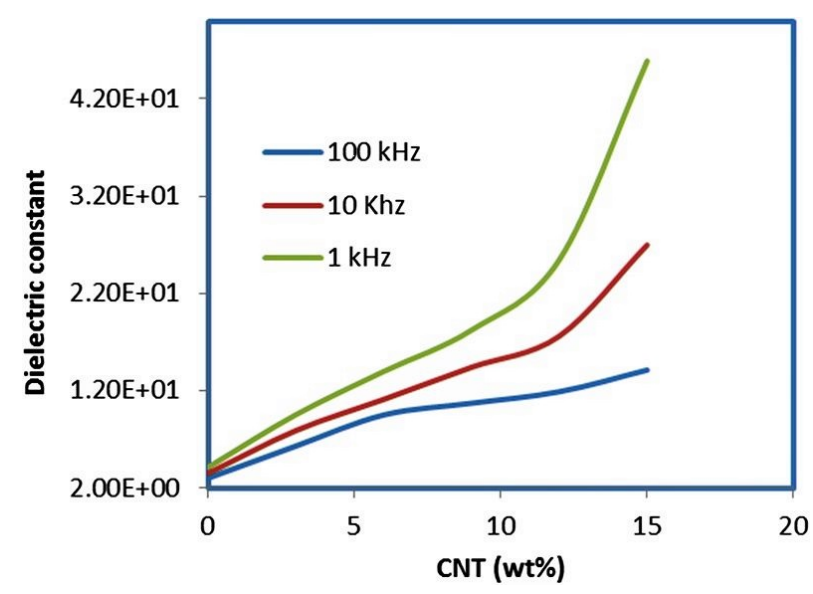

Fig. 2. Dielectric constant change of the polyimide/MWCNTs composite films as a function of CNTs content at different frequencies.

The current-voltage characteristics displayed in Fig. 3 show that at MWCNTs loadings in excess of 3 wt.\%, nonlinear I-V relationships are observed. This non-ohmic behavior is most likely due to a tunneling mechanism.

Conduction may occur by electron hopping from one nanotube to an adjacent one when they 


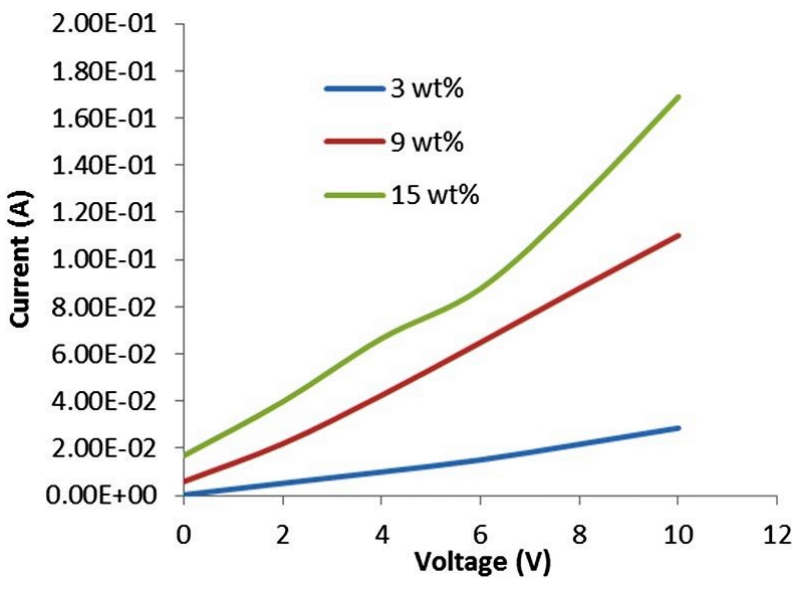

Fig. 3. DC current-voltage behavior of MWCNTs/PI nanocomposites at three different concentrations of MWCNTs.

are close enough. Above percolation, conductive paths are formed through the composite due to quantum tunneling effect when the distance between the conductive inclusions is such that electron hopping can occur. Percolation theory assumes that the paths are made of conductive inclusions in direct contact. However, in the case of quantum tunneling, a contact resistance exists within the conductive path, between two adjacent MWCNTs [13, 14].

The microscope images of the polyimide/MWCNT nanocomposite films are shown in Fig. 4. The surface of the pure PI is generally flat, but the surface of the PI/MWCNTs nanocomposites is comparatively rough. The dispersion of MWCNTs in the polyimide matrix seems to be more uniform. The rough surface could have been caused by the strong interfacial interaction between the MWCNTs and the PI [15].

The greatly improved dispersion of MWCNTs in the PI matrix might be due to the strong interfacial interactions (covalent and hydrogen bonds) as well as to the chemical compatibility between the PI matrix and the modified MWCNTs. However, MWCNTs incorporated in the PI matrix exist as individual tubes or small nanotube bundles. Modified MWCNTs are well dispersed without any obvious aggregation $[6,16]$.

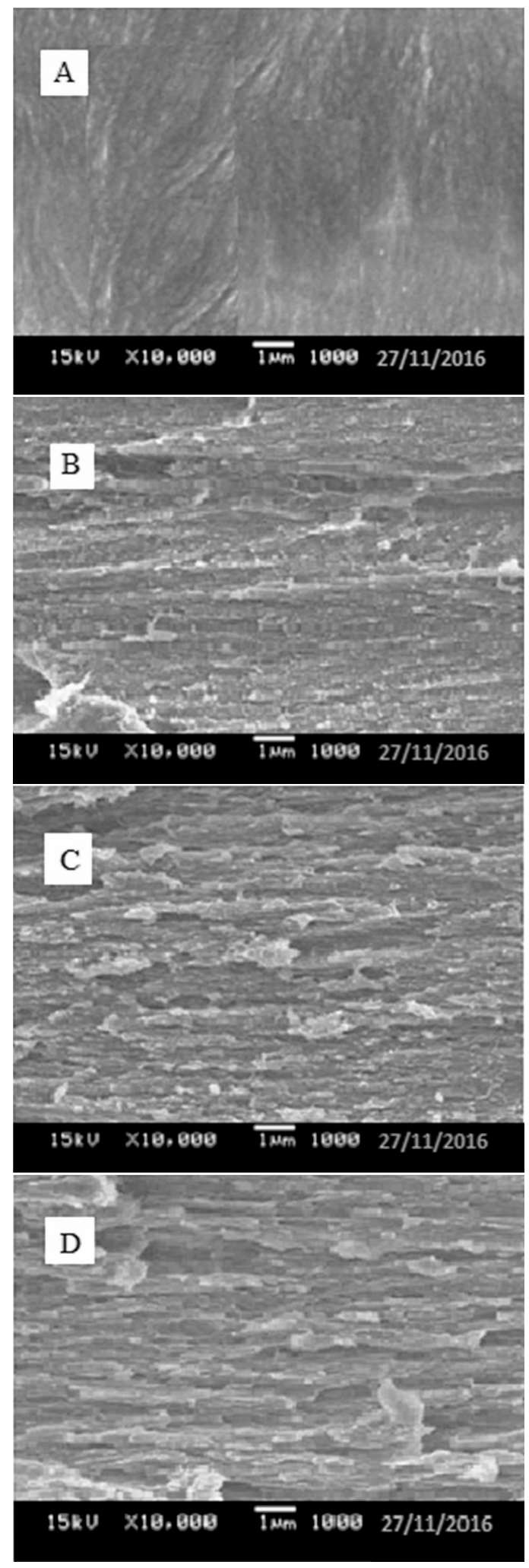

Fig. 4. SEM images of (A) pure PI, (B) PI/3 wt. $\%$ MWCNT, (C) PI/9 wt.\% MWCNT, (D) PI/15 wt.\% MWCNT nanocomposites. 


\section{Conclusions}

Polyimide/MWCNTs composites were prepared using an efficient solution process. Electrical properties of MWCNTs reinforced polyimide composites were studied using both $\mathrm{AC}$ and DC measurement techniques. The PI polymers gradually transformed from an insulator to a conductive system at a particular percolation threshold at and above 9 wt.\% MWCNTs. It was observed that the electrical conductivity and dielectric constants enhanced significantly by several orders of magnitude up to $15 \mathrm{wt} \%$ of MWCNTs incorporation. The electrical conductivity increase with the frequency can be attributed to high dislocation density near the interface.

The high dielectric constant at a low frequency of $1 \mathrm{kHz}$ is thought to originate from the space charge polarization mechanism. I-V characteristics indicate a significant increase in current with an increase in MWCNTs concentration in the composites. The dispersion of MWCNTs in the PI matrix has been achieved, as evidenced by SEM observations.

\section{References}

[1] Wei B.Q., Vajtai R., Ajayan P.M., Appl. Phys. Lett., 79 (2001), 1172.

[2] Upadhyay A.N., Tiwari R.S., Singh K., $A d v$. Mater. Lett., 6 (2015), 1098.
[3] Khare R., Bose S., J. Miner. Mater. Character. Eng., 4 (2005), 31

[4] Nayak L., Rahaman M., Aldalbahi A., Chaki T.K., Khastgir D., Poly. Eng. Sci., (2016). DOI 10.1002/pen.24412.

[5] Zhang Q., Li J., Zhao X., Chen D., Poly. Int., 58 (2009), 557.

[6] Chen Y., Lin B., Zhang X., Wang J., Lai Ch., Sun Y., LiUab Y., Yanga H., J. Mater. Chem., 2 (2014), 14118.

[7] Park S., Chae S., Rhee J., Kang S., Bull. Korean Chem. Soc., 31 (2010), 2279.

[8] Mo T., Wang H., Chen S., Yeh Y., Poly. Compos., 29 (2008), 451.

[9] Jiang X., Bin Y., Matsuo M., Poly., 46 (2005), 7418.

[10] Thuau D., Koutsos V., Cheung R., J. Vac. Sci. Technol., 27 (2009), 3139.

[11] Ounaies Z., Park C., Wise K.E., Siochi E.J., Harrison J.S., Comp. Sci. Technol., 63 (2003), 1637.

[12] Zhang Y., Yu L., Zhao L., Tong W., Huang H., Ke S., Chan H. L. W., J. Electron. Mater., 41 (2012), 2281.

[13] KIM B.S., Macromol. Res., 15 (2007), 357.

[14] Yuen S., Ma C. M., Chiang C., Lin Y., Teng C., J. Poly. Sci. Poly. Chem., 45 (2007), 3349.

[15] QU L., LiN Y., Hill D. E., ZHOU B., WANG W., Sun X., KitaygorodskiY A., Suarez M., ConNell J. W., Allard L. F., Sun Y., Macromol., 37 (2004), 6055.

[16] Lim J., Shin D.G., Yeo H., Goh M., Ku B., Yang C., Lee D.S., Hwang J., Park B., You N., J. Poly. Sci. Poly. Phys., 52 (2014), 960.

Received 2016-11-29

Accepted 2017-11-06 